PROCEEDINGS OF THE

AMERICAN MATHEMATICAL SOCIETY

Volume 127, Number 8, Pages 2395-2403

S 0002-9939(99)05034-0

Article electronically published on April 15, 1999

\title{
UNIFORM WEAK IMPLIES UNIFORM STRONG PERSISTENCE FOR NON-AUTONOMOUS SEMIFLOWS
}

\author{
HORST R. THIEME
}

(Communicated by Hal L. Smith)

\begin{abstract}
It is shown that, under two additional assumptions, uniformly weakly persistent semiflows are also uniformly strongly persistent even if they are non-autonomous. This result is applied to a time-heterogeneous model of S-I-R-S type for the spread of infectious childhood diseases. If some of the parameter functions are almost periodic, an almost sharp threshold result is obtained for uniform strong endemicity versus extinction.
\end{abstract}

\section{INTRODUCTION}

Persistence (or permanence) is an important property of dynamical systems and of the systems in ecology, epidemics, etc. they are modeling. On the one hand it is an important concept in itself addressing the long-term survival of some or all components of a system: see Hutson, Schmitt [16] and Waltman [26], for reviews and references of the development until the early nineties, and Zhao [28] for an update. On the other hand, persistence is also an essential intermediate result leading to more precise statements concerning the systems' qualitative behavior, e.g., the existence or global stability of an equilibrium: see, e.g., Lin, So [17], Feng, Thieme [8], Section 4, Zhao [28, 30], Smith, Waltman [21]. Persistence becomes the more relevant, the more complex the systems become, so that little else can be said about its large-time behavior by analytic methods. While this trend can be observed for systems of autonomous partial differential equations (e.g., modeling spatially heterogeneous ecological systems, Cantrell et al. [5], or competition of randomly moving microbial species in a flow reactor, Ballyk et al. [1], persistence becomes even more important (and difficult) for non-autonomous dynamical systems (semiflows) where obvious candidates for the long-time behavior of the system (equilibria, periodic orbits) are usually missing.

Freedman, Moson [10] discovered that uniform weak persistence implies uniform (strong) persistence for autonomous semiflows. Thieme [23] elaborates that this is not only an interesting theoretical result, but also a very useful tool to establish uniform persistence because uniform weak persistence can conveniently be shown by the method of fluctuation, by acyclicity of the boundary flow, or by ad hoc methods. Another condition under which (not necessarily uniform) weak persistence implies uniform strong persistence is presented by Freedman, Ruan, Tang [11].

Received by the editors November 10, 1997.

1991 Mathematics Subject Classification. Primary 34C35, 34D05, 92D30.

The author's research was partially supported by NSF grant DMS-9403884.

(C)1999 American Mathematical Society 
In this note we show that uniform weak persistence implies uniform strong persistence also for non-autonomous semiflows (Section 2). The result is not so elegant as in the autonomous case, as we will need extra conditions. That such additional conditions, unpleasant as they are, may be natural becomes evident once one tries to reduce the non-autonomous result to the autonomous case by using skew product flows (see Hale [13], e.g., for the latter). We do not take this approach here because a general skew product flow acts both on the original space and on translations of the original non-autonomous system, and looking for a useful metric topology on the latter is a hassle we want to avoid. This difficulty does not arise when nonautonomous ordinary differential equations are considered where the skew product flow can operate on translations of the vector field (Gatica, So, [12]; Burton, Hutson [3]). We prefer to work directly with the non-autonomous semiflow adapting the approach in Thieme [23]. This is a topological approach in spirit, an alternative being the use of (vector) Lyapunov-type functions (e.g., Burton, Hutson [3]; Tang, Kuang [22]). Actually Lyapunov-type functions may also be useful in establishing uniform weak persistence. We hope to narrow the gap between the two approaches by introducing the concept of persistence with respect to a functional $\rho$; traditionally $\rho(x)$ is the distance of the point $x$ from the 'boundary'.

At this point it is not clear (except for the special cases of asymptotically autonomous or periodic semiflows) whether the boundary flow analysis (using the Butler-McGehee lemma), which can be so powerful in the autonomous or periodic case with its acyclicity conditions (see Hale, Waltman [14]; Thieme [23]; Yang, Ruan [27]; Zhao [28], e.g.), can be extended to non-autonomous semiflows. Typically one will rely on an ad hoc proof by contradiction, sometimes supported by the method of fluctuations, to establish uniform weak persistence. What kind of results one may obtain this way is illustrated for a time-heterogeneous S-I-R-S type model for the spread of infectious childhood diseases (Section 3). In the case that some of the parameter functions are almost periodic we establish an almost sharp threshold result for the disease to be uniformly strongly endemic (i.e., to be uniformly strongly persistent in the population). This result involves a threshold parameter which, mathematically, plays the role of a basic replacement ratio, but does not necessarily have the same epidemiologic explanation.

\section{UNIFORM WEAK IS UNIFORM STRONG}

Taking a topological point of view, we let $(X, d)$ be a metric space,

$$
\Delta=\left\{(t, s) ; r_{0} \leq s \leq t<\infty\right\}, \quad r_{0} \in \mathbf{R} .
$$

A mapping $\Psi: \Delta \times X \rightarrow X$ is called a (non-autonomous) semiflow on $X$ (anchored at $\left.r_{0}\right)$ if

$$
\Psi(t, s, \Psi(s, r, x))=\Psi(t, r, x), \quad \Psi(r, r, x)=x \quad \forall t \geq s \geq r \geq r_{0}, x \in X .
$$

If the mapping $\Psi$ is continuous and a semiflow, it is called a continuous semiflow. $\Psi$ is called an autonomous semiflow if $\Psi(t+r, r, x)$ does not depend on $r \geq r_{0}$ for $t \geq 0, x \in X$. Let

$$
\rho: X \rightarrow(0, \infty)
$$

be a continuous strictly positive functional on $X$. We consider the function

$$
\sigma:[0, \infty) \times X \times\left[r_{0}, \infty\right) \rightarrow(0, \infty)
$$


defined by

$$
\sigma(t, x ; r)=\rho(\Psi(t+r, r, x)), \quad t \geq 0, x \in X, r \geq r_{0} .
$$

We notice the following relation between $\sigma$ and $\Psi$ :

$$
\begin{aligned}
\sigma(t, \Psi(s, r, x) ; s) & =\rho(\Psi(t+s, s, \Psi(s, r, x))=\rho(\Psi(t+s, r, x)) \\
& =\sigma(t+s-r, x ; r) .
\end{aligned}
$$

We introduce the following notation:

$$
\sigma^{\infty}(x, r)=\limsup _{t \rightarrow \infty} \sigma(t, x ; r), \quad \sigma_{\infty}(x, r)=\liminf _{t \rightarrow \infty} \sigma(t, x ; r) .
$$

Definition 2.1. $\Psi$ is called weakly $\rho$-persistent if $\sigma^{\infty}(x, r)>0 \quad \forall x \in X, r \geq r_{0}$.

$\Psi$ is called strongly $\rho$-persistent if $\sigma_{\infty}(x, r)>0 \quad \forall x \in X, r \geq r_{0}$.

$\Psi$ is called uniformly weakly $\rho$-persistent if there exists some $\epsilon>0$ such that

$$
\sigma^{\infty}(x, r)>\epsilon \quad \forall x \in X, r \geq r_{0} .
$$

$\Psi$ is called uniformly strongly $\rho$-persistent if there exists some $\epsilon>0$ such that

$$
\sigma_{\infty}(x, r)>\epsilon \quad \forall x \in X, r \geq r_{0} .
$$

If no misunderstanding about the functional $\rho$ is possible, we use persistent rather than $\rho$-persistent.

$\Psi$ is said to have property (CA) if the following holds:

(CA) There exists some $\epsilon_{0}>0$ and a closed subset $B$ in $X$ with the following properties:

- For all $x \in\left\{\rho \leq \epsilon_{0}\right\}, s \geq r_{0}$, we have $\Psi(t, s, x) \rightarrow B, \quad t \rightarrow \infty$.

- The intersections of $B$ with the rings $\{\alpha \leq \rho \leq \beta\}, 0<\alpha<\beta<\infty$, have compact closure in $X$.

We call the set $B$ in (CA) an attractor for $\Psi$. By $\Psi(t, s, x) \rightarrow B$ as $t \rightarrow \infty$, we mean that for every open set $U$ containing $B$, there exists some $t_{U}>0$ such that $\Psi(t, s, x) \in U$ for all $t>t_{U}$. Equivalently the distance of $\Psi(t, s, x)$ from $B$ tends to 0 as $t \rightarrow \infty$.

For every $x \in B$ we define sets $\Sigma(x)$ as follows:

$$
\begin{array}{ll}
\Sigma(x) \quad \text { consists of functions } \quad \bar{\sigma}_{x}:[0, \infty) \rightarrow \mathbf{R}, \\
\bar{\sigma}_{x}(t)=\lim _{j \rightarrow \infty} \sigma\left(t, x ; s_{j}\right) \quad \text { locally uniformly in } t \geq 0, \\
& \text { for some sequence }\left(s_{j}\right) \text { in }\left[r_{0}, \infty\right) \text { with } s_{j} \rightarrow \infty \text { as } j \rightarrow \infty .
\end{array}
$$

The sets $\Sigma(x)$ may be empty.

Theorem 2.2. Let $\Psi$ be a semiflow that is uniformly weakly $\rho$-persistent and has the property (CA) with attractor $B$.

Further assume that $\sigma(\cdot, \cdot ; s)$ is continuous on $[0, \infty) \times B$, uniformly in $s \geq r_{0}$. Finally assume that

$$
\liminf _{s \rightarrow \infty} \sigma(t, x ; s)>0 \quad \forall t \geq 0, x \in B
$$

and that there exists some $\epsilon>0$ such that

$\bullet_{2} \quad \limsup _{t \rightarrow \infty} \bar{\sigma}_{x}(t)>\epsilon \quad \forall x \in B, \bar{\sigma}_{x} \in \Sigma(x)$.

Then $\Psi$ is uniformly strongly persistent.

If the semiflow $\Psi$ is autonomous, $\sigma(\cdot ; s)$ is independent of $s$ and $\bar{\sigma}_{x}(t)=\sigma(t, x ; 0)$. So the two extra assumptions are automatically satisfied. 
Proof. Assume that $\Psi$ is uniformly weakly persistent. Then there exists $\epsilon>0$ such that

$$
\sigma^{\infty}(x, r)>\epsilon \quad \forall x \in X, r \geq r_{0} .
$$

We can choose $\epsilon$ smaller than $\epsilon_{0}$ in the compactness assumption (CA). We can also arrange that this $\epsilon$ satisfies the assumption $\bullet_{2}$ in the theorem.

Step 1: Let $x \in X$ and $r \geq r_{0}$ such that $\liminf _{t \rightarrow \infty} \rho(\Psi(t, r, x))<\epsilon_{1}<\epsilon$.

Then there exist sequences $\left(s_{k}\right)$ in $\left[r_{0}, \infty\right)$ and $\left(t_{k}\right)$ in $(0, \infty)$ such that $s_{k} \rightarrow \infty$ $(k \rightarrow \infty)$ and

$$
\begin{aligned}
& \rho\left(\Psi\left(s_{k}, r, x\right)\right)=\epsilon, \quad \rho\left(\Psi\left(t_{k}+s_{k}, r, x\right)\right)<\epsilon_{1} \quad \forall k \in \mathbf{N}, \\
& \rho\left(\Psi\left(s+s_{k}, r, x\right)\right) \leq \epsilon, \quad \text { whenever } 0 \leq s \leq t_{k} .
\end{aligned}
$$

Moreover, by the first part of our compactness assumption (CA),

$$
\Psi\left(s_{k}, r, x\right) \rightarrow B, \quad k \rightarrow \infty .
$$

Hence we find elements $b_{k} \in B$ such that

$$
d\left(\Psi\left(s_{k}, r, x\right), b_{k}\right) \rightarrow 0, \quad k \rightarrow \infty .
$$

Since $\rho$ is continuous, the elements $b_{k}$ are in the intersection of $B$ with the ring $\left\{\epsilon / 2<\rho<\epsilon_{0}\right\}$ for sufficiently large $k$. After dropping the first elements from the sequence we can assume that

$$
b_{k} \in K:=B \cap\left\{\epsilon / 2 \leq \rho \leq \epsilon_{0}\right\} \quad \forall k \in \mathbf{N} .
$$

The set $K$ has compact closure by the second part of assumption (CA).

Step 2: Suppose that $\Psi$ is not uniformly strongly persistent.

Then there exist sequences $\left(r_{j}\right)$ in $\left[r_{0}, \infty\right)$ and $\left(x_{j}\right) \in X$ such that

$$
\liminf _{t \rightarrow \infty} \rho\left(\Psi\left(t, r_{j}, x_{j}\right)\right) \rightarrow 0, \quad j \rightarrow \infty .
$$

Using step 1 and a diagonalization procedure we obtain sequences $\left(r_{j}\right)$ and $\left(s_{j}\right)$ in $\left[r_{0}, \infty\right)$, and $\left(t_{j}\right)$ in $[0, \infty)$ such that $s_{j}>r_{j}, s_{j} \rightarrow \infty$ as $j \rightarrow \infty$, and

$$
\begin{aligned}
& \rho\left(\Psi\left(s_{j}, r_{j}, x_{j}\right)\right)=\epsilon, \\
& \rho\left(\Psi\left(t_{j}+s_{j}, r_{j}, x_{j}\right)\right) \rightarrow 0, \quad j \rightarrow \infty . \\
& \rho\left(\Psi\left(s+s_{j}, r_{j}, x_{j}\right)\right) \leq \epsilon, \quad 0 \leq s \leq t_{j} .
\end{aligned}
$$

Further we find elements $b_{j}$ in the relatively compact set $K$ such that

$$
d\left(b_{j}, \Psi\left(s_{j}, r_{j}, x_{j}\right)\right) \rightarrow 0, \quad j \rightarrow \infty .
$$

The sequence $\left(b_{j}\right)$ has an accumulation point $x \in \bar{K} \subseteq B$. After choosing subsequences we can assume that

$$
\Psi\left(s_{j}, r_{j}, x_{j}\right) \rightarrow x, \quad j \rightarrow \infty .
$$

We claim: $t_{j} \rightarrow \infty$ as $j \rightarrow \infty$.

If not, $t_{j} \rightarrow t$ after choosing subsequences. Then, by the definition of $\sigma$ in (2.1),

$$
\begin{aligned}
0 & =\limsup _{j \rightarrow \infty} \rho\left(\Psi\left(t_{j}+s_{j}, r_{j}, x_{j}\right)\right)=\limsup _{j \rightarrow \infty} \rho\left(\Psi\left(t_{j}+s_{j}, s_{j}, \Psi\left(s_{j}, r_{j}, x_{j}\right)\right)\right) \\
& =\limsup _{j \rightarrow \infty} \sigma\left(t_{j}, \Psi\left(s_{j}, r_{j}, x_{j}\right) ; s_{j}\right) .
\end{aligned}
$$


Since $\sigma(\cdot ; s)$ is continuous uniformly in $s$, we have

$$
0=\limsup _{j \rightarrow \infty} \sigma\left(t, x ; s_{j}\right)
$$

which contradicts $\bullet_{1}$ because $x \in B$.

Let $t \geq 0$ now be arbitrary. Since $t_{j} \rightarrow \infty$ as $j \rightarrow \infty, t_{j}>t$ for sufficiently large $j$. Hence

$$
\epsilon \geq \limsup _{j \rightarrow \infty} \rho\left(\Psi\left(t+s_{j}, r_{j}, x_{j}\right)\right)=\limsup _{j \rightarrow \infty} \sigma\left(t, \Psi\left(s_{j}, r_{j}, x_{j}\right) ; s_{j}\right) .
$$

Using again that $\sigma(\cdot ; s)$ is uniformly continuous in $s \geq r_{0}$,

$$
\epsilon \geq \limsup _{j \rightarrow \infty} \sigma\left(t, x ; s_{j}\right),
$$

where $x$ is the limit of $\Psi\left(s_{j}, r_{j}, x_{j}\right), x \in B$. This implies that, for every $t \geq 0$, the sequence $\left(\sigma\left(t, x ; s_{j}\right)\right)$ is bounded. Since $\sigma(\cdot, x ; s)$ is continuous on $[0, \infty)$ uniformly in $s$, by the Arzela-Ascoli theorem, there exists a function $\bar{\sigma}_{x}$ on $[0, \infty)$ such that, after choosing a subsequence,

$$
\sigma\left(t, x ; s_{j}\right) \rightarrow \bar{\sigma}_{x}(t), \quad j \rightarrow \infty \text {, locally uniformly in } t \geq 0 .
$$

So we have found $x \in B$ and a function $\bar{\sigma}_{x} \in \Sigma(x)$ such that

$$
\epsilon \geq \bar{\sigma}_{x}(t) \quad \forall t \geq 0
$$

which contradicts $\bullet$.

In order to become more familiar with the role of the set $\Sigma$ in (2.3) we consider asymptotically autonomous semiflows. We recall that a semiflow $\Psi$ is called asymptotically autonomous if there exists an autonomous semiflow $\Phi$ such that, for all sequences $t_{j} \rightarrow t, s_{j} \rightarrow \infty, x_{j} \rightarrow x$ as $j \rightarrow \infty$,

$$
\Psi\left(t_{j}+s_{j}, s_{j}, x_{j}\right) \rightarrow \Phi(t, x), \quad j \rightarrow \infty .
$$

$\Phi$ is called the limit flow associated with $\Psi$.

First, this implies that $\sigma(t, x ; s)=\rho(\Psi(t, s, x))$ is continuous in $(t, x)$ uniformly in $s \geq r_{0}$. Secondly, since, for any sequences $t_{j} \rightarrow t, x_{j} \rightarrow x, s_{j} \rightarrow \infty$ as $j \rightarrow \infty$, we have

$$
\sigma\left(t_{j}, x_{j} ; s_{j}\right) \rightarrow \rho(\Phi(t, x)), \quad j \rightarrow \infty,
$$

for every $x \in X, \Sigma(x)$ has exactly one element $\bar{\sigma}_{x}$, namely $\bar{\sigma}_{x}(t)=\rho(\Phi(t, x))$.

The following result is now immediate from Theorem 2.2 .

Corollary 2.3. Let $\Psi, \Phi$ be continuous semiflows on $X$ that are uniformly weakly $\rho$-persistent. Let (CA) holds for $\Psi$ with an attractor $B$. Further assume that $\Phi$ is autonomous, $\Psi$ is asymptotically autonomous, and $\Phi$ the limit semiflow of $\Psi$. Then $\Psi$ is uniformly strongly $\rho$-persistent.

We remark that uniform strong $\rho$-persistence of the limit flow $\Phi$ is not sufficient even for weak $\rho$-persistence of the asymptotically autonomous semiflow $\Psi$. (See Section 2.1 of Thieme [24].)

Similarly, one can explicitly determine $\Sigma(x)$, if the semiflow $\Psi$ is periodic or asymptotically periodic. We leave it to the reader to derive corollaries like Corollary 2.3 for these special non-autonomous semiflows from Theorem 2.2. For related material we refer to Zhao $([28,29,30])$. 
Traditionally, the topologically oriented school of persistence theory assumes that $X$ is the open subset of a larger metric space $Z$ and that $\rho(x), x \in X$, is the distance of $x$ from $Z \backslash X$. Looking at some general functional $\rho$ earns us some extra flexibility as demonstrated by the following Corollary which, for simplicity, we only state in the special case of an asymptotically autonomous semiflow in a finite dimensional space.

Corollary 2.4. Let $X$ be a closed subset of $\mathbf{R}^{n}$ and $\Psi, \Phi$ be continuous semiflows on $X$. Further assume that $\Phi$ is autonomous, $\Psi$ is asymptotically autonomous, and $\Phi$ the limit semiflow of $\Psi$.

Finally assume that there exists some $c>0$ such that

$$
\liminf _{t \rightarrow \infty}\|\Phi(t, x)\|<c \quad \forall x \in X
$$

and

$$
\liminf _{t \rightarrow \infty}\|\Psi(t, s, x)\|<c \quad \forall x \in X, s \geq r_{0} .
$$

Then there exists some $c>0$ such that

$$
\limsup _{t \rightarrow \infty}\|\Psi(t, s, x)\|<c \quad \forall x \in X, s \geq r_{0} .
$$

Proof. Set $\rho(x)=(1+\|x\|)^{-1}$. The compactness condition (CA) is trivially satisfied by choosing $B=X$ because the $\rho$-shells are compact. Obviously $\Phi$ and $\Psi$ are weakly uniformly $\rho$-persistent. So $\Psi$ is strongly uniformly $\rho$-persistent by Corollary 2.3 .

\section{The time-Heterogeneous S-I-R-S Model}

As an application of the theory developed in the previous section, we consider a model for the spread of infectious childhood diseases. It has been argued that the school system induces a time-heterogeneity in the per capita/capita infection rate, $\alpha$, because the chain of infections is interrupted or at least weakened by the vacations and new individuals are recruited into a scene with higher infection risk at the beginning of each school year (Schenzle [18]; Dietz, Schenzle [7]). Here we consider a model without exposed period; the total population, with size $N$, is divided into its susceptible, $S$, infective, $I$, and recovered, $R$, parts, and the contraction of the disease is modeled by the law of mass action involving susceptibles and infectives:

$$
\begin{aligned}
N & =S+I+R, \\
\frac{d}{d t} I & =-\mu(t) I+\alpha(t) S I-\gamma(t) I, \\
\frac{d}{d t} R & =-\mu(t) R+\gamma(t) I-\eta(t) R .
\end{aligned}
$$

We assume that the disease causes no fatalities and that the population size $N(t)$ is a given function of time $t . \mu(t)$ is the instantaneous per capita mortality rate. $\gamma(t)$ and $\eta(t)$ are the instantaneous per capita rates of leaving the infective stage or removed stage, respectively.

The case that $\alpha$ is a periodic function and that $N, \mu, \gamma$ and $\eta$ are constant has been extensively studied, see Dietz, Schenzle [7] and Hethcote, Levin [15] for reviews and references. We mention that periodic solutions can exist whose periods 
are integer multiples of the period of $\alpha$ and that co-existence of stable periodic solutions with different periods is possible (Smith [19, 20]).

Here we assume that $N, \mu, \alpha, \gamma$, and $\eta$ are non-negative bounded continuous functions. Further we assume that $N, \mu, \alpha$ and $\gamma$ are strictly positive and almost periodic. $\eta$ is not assumed to be almost periodic and the solutions of (3.1) will not necessarily be almost periodic as a consequence (cf. Gatica, So [12]). We will state an almost sharp threshold result for uniform strong persistence of the disease versus disease extinction. Detailed proofs will be given elsewhere (Thieme [25]), here we will only give some explanations. Unfortunately our approach does not seem to extend to the S-E-I-R-S model.

We introduce

$$
\mathcal{R}_{\diamond}=\frac{\overline{\alpha N}}{\bar{\mu}+\bar{\gamma}}, \quad \overline{\alpha N}=\lim _{t \rightarrow \infty} \frac{1}{t} \int_{0}^{t} \alpha(s) N(s) d s, \quad \bar{\gamma}=\lim _{t \rightarrow \infty} \frac{1}{t} \int_{0}^{t} \gamma(s) d s,
$$

and $\bar{\mu}$ analogously to $\bar{\gamma}$. The limits of the time averages exist because the integrands are almost periodic. The following threshold result has been phrased in terms of the number $\mathcal{R}_{\diamond}$ which formally plays the role of a basic replacement ratio without having this interpretation. Notice that in general $\mathcal{R}_{\diamond}$ is not the asymptotic time average of the instantaneous basic replacement ratios

$$
\mathcal{R}_{0}(t)=(\alpha(t) N(t)) /(\mu(t)+\gamma(t)) .
$$

Theorem 3.1. (a) If $\mathcal{R}_{\diamond}<1$, the disease dies out, i.e., for every solution of (3.1) we have $I(t), R(t) \rightarrow 0$ as $t \rightarrow \infty$.

(b) If $\mathcal{R}_{\diamond}>1$, the disease persists uniformly strongly in the population in the sense that there exists some $\epsilon>0$ such that $\liminf _{t \rightarrow \infty} I(t)>\epsilon$ for all solutions of (3.1) with $I(0)>0, R(0) \geq 0$.

Part (a) can be proved in a straightforward way by considering $\ln I(t)$. In part (b) uniform weak persistence can be shown by a contradiction argument again considering $\ln I(t)$. For both disease extinction and uniform weak disease persistence, the almost periodicity of the various functions is only needed in so far as it guarantees the convergence of the time averages. Almost periodicity or a similar property is needed, however, to satisfy condition $\bullet_{2}$ in Theorem 2.2 from which Theorem 3.1 will follow. In order to find a sufficient condition for uniform strong persistence, the combination of almost periodicity with $\mathcal{R}_{\diamond}>1$ can be replaced by a condition similar to Burton, Hutson [3]), Theorem 4.2. In order to apply Theorem 2.2, we choose as metric space $X=\{(I, R) ; I>0, R \geq 0\}$ and $\rho(I, R)=I$ as functional. Property (CA) is satisfied because $I^{\infty} \leq \sup N$ and $R^{\infty} \leq \frac{\sup \gamma}{\inf \mu} I^{\infty}$ with $I^{\infty}, R^{\infty}$ denoting the limit superior of $I, R$ respectively.

In order to keep the illustration short, we have chosen an ODE model, and Theorem 3.1 can presumably be also proved using an average Lyapunov function like in Burton, Hutson [3] where average type conditions guarantee predator persistence in a prey predator system. However, since Theorem 2.2 does not only hold for ordinary differential equations, but for general semiflows, Theorem 3.1 holds under the same assumptions for $N, \mu, \alpha, \gamma$, if the exponential distribution of the length of the removed stage, described by $\eta$, is replaced by an arbitrary distribution. Details will be presented elsewhere (Thieme [25]); see Castillo-Chavez, Thieme [6] for the model modification. 


\section{ACKNOWLEDGEMENT}

Thanks to Hal Smith for rekindling my interest in persistence and to Xiao-Qiang Zhao for bibliographic hints.

\section{REFERENCES}

1. Ballyk, M.; Dung, Le; Jones, D.A.; Smith, H.L. (to appear): Effect of random motility on microbial growth and competition in a flow reactor. SIAM J. Appl. Math.

2. Bremermann, H.J.; Thieme, H.R. (1989): A competitive exclusion principle for pathogen viruses. J. Math. Biol. 27, 179-190 MR 90d:92021

3. Burton, T.A.; Hutson, V. (1991): Permanence for non-autonomous predator-prey systems. Diff. Integral Equations 4, 1209-1280 MR 93f:34019

4. Busenberg, S.; Cooke, K.L.; Thieme, H.R. (1991): Demographic change and persistence of HIV/AIDS in a heterogeneous population. SIAM J. Appl. Math. 51, 1030-1052 MR 92j:92016

5. Cantrell, R.S.; Cosner, C.; Hutson, V. (1996): Ecological models, permanence and spatial heterogeneity. Rocky Mountain J. Math 26, 1-35 MR 97g:92011

6. Castillo-Chavez, C.; Thieme, H.R. (1995) Asymptotically autonomous epidemic models. Mathematical Population Dynamics: Analysis of Heterogeneity I. Theory of Epidemics (O. Arino et al., eds.) Wuerz, 33-50.

7. Dietz, K.; Schenzle, D. (1985): Mathematical models for infectious disease statistics. A Celebration of Statistics. The ISI Centenary Volume ed. by A.C. Atkinson, S.E. Fienberg, 167-204. Springer MR 87h:92054

8. Feng, Z.; Thieme, H.R. (1995): Recurrent outbreaks of childhood diseases revisited: the impact of isolation. Math. Biosci. 128, 93-130 MR 96b:92010

9. Fernandes, M.L.C. (1990): Uniform repellers for processes with applications to periodic differential systems. J. Diff. Equations 86, 141-157 MR 91g:58165

10. Freedman, H.I.; Moson, P. (1990): Persistence definitions and their connections. Proc. AMS 109, 1025-1032 MR 90k:34054

11. Freedman, H.I.; Ruan, S.; Tang, M. (1994): Uniform persistence and flows near a closed positively invariant set. J. Dynamics Diff. Equations 6, 583-600 MR 95i:34080

12. Gatica, J.A.; So, J. W.-H. (1988): Predator-prey models with almost periodic coefficients. Appl. Anal. 27, 143-152 MR 89a:92057

13. Hale, J.K. (1989): Asymptotic Behavior of Dissipative Systems. AMS MR 89g:58059

14. Hale, J.K.; Waltman, P. (1989): Persistence in infinite-dimensional systems. SIAM J. Math. Anal. 20, 388-395 MR 90b:58156

15. Hethcote, H.W.; Levin, S.A. (1989): Periodicity in epidemiological models. Applied Mathematical Ecology (S.A. Levin, T.G. Hallam, L.J. Gross; eds.), 193-211. Biomathematics 18, Springer CMP 90:04

16. Hutson, V.; Schmitt, K. (1992): Permanence in dynamical systems. Math. Biosci. 111, 1-71 MR 93d:92003

17. Lin, X.; So, J. W.-H. (1993): Global stability of the endemic equilibrium and uniform persistence in epidemic models with subpopulations. J. Austral. Math. Soc., Ser. B, 34, 282-295 MR 93k:92010

18. Schenzle, D. (1984): An age-structured model of pre- and postvaccination measles transmission. IMA J. Math. Appl. Med. Biol. 1, 169-191 CMP 18:16

19. Smith, H.L. (1983a): Subharmonic bifurcation in an SIR epidemic model. J. Math. Biol. 17, 163-177 MR 85d:92033a

20. Smith, H.L. (1983b): Multiple stable subharmonics for a periodic epidemic model. J. Math. Biol. 17, 179-190 MR 85d:92033b

21. Smith, H.L.; Waltman, P. (to appear): Perturbation of a globally stable steady state. Proc. Amer. Math. Soc. CMP 98:05

22. Tang, B.; Kuang, Y. (1996): Permanence in Kolmogorov-type systems of nonautonomous functional differential equations. J. Math. Anal. Appl. 197, 427-447 MR 96k:34164

23. Thieme, H.R. (1993): Persistence under relaxed point-dissipativity (with applications to an epidemic model). SIAM J. Math. Anal. 24, 407-435 MR 94a:34055

24. Thieme, H.R. (1994): Asymptotically autonomous differential equations in the plane. Rocky Mountain J. Math. 24, 351-380 MR 96a:34095

25. Thieme, H.R. (preprint): Persistence and permanence for non-autonomous semiflows 
26. Waltman, P. (1991): A brief survey of persistence in dynamical systems. Delay Differential Equations and Dynamical Systems (S. Busenberg and M. Martelli, eds.), 31-40. Lecture Notes in Mathematics 1475. Springer MR 92j:34093

27. Yang, F.; Ruan, S. (1996): A generalization of the Butler-McGehee lemma and its applications in persistence theory. Diff. Integral Equations 9, 1321-1330 CMP 97:01

28. Zhao, X.-Q. (1995): Uniform persistence and periodic coexistence states in infinitedimensional periodic semiflows with applications. Canad. Appl. Math. Quart. 3, 473-495 MR 96k:58188

29. Zhao, X.-Q. (1996): Asymptotic behavior for asymptotically periodic semiflows with applications. Comm. Appl. Nonl. Anal. 3, 43-66 MR 97i:58150

30. Zhao, X.-Q. (1997): Global asymptotic behavior in a periodic competitor-competitormutualist parabolic system. Nonlinear Anal., TMA 29, 551-568 CMP 97:13

Department of Mathematics, Arizona State University, Tempe, Arizona 85287-1804

E-mail address: h.thieme@asu.edu 